Quality and Quantity, 17 (1983) 387-404

Elsevier Science Publishers B.V., Amsterdam - Printed in The Netherlands

\title{
Analysis of Preferences as Directional Data
}

\section{CHARLES L. JONES}

Sociology Department, 563 Spadina Avenue

University of Toronto, Toronto, Ontario, Canada 


\title{
Analysis of Preferences as Directional Data
}

\author{
CHARLES L. JONES
}

Sociology Department, 563 Spadina Avenue, University of Toronto, Toronto, Ontario, Canada

Continuous data are normally thought of as being on a linear continuum. However, there are some fields in which important aspects of phenomena are captured by treating data as directions: for example, as points on the circumference of a unit circle, or equivalently as angles between $0^{\circ}$ and $360^{\circ}$. Such data require careful treatment, for any transformation of angular data must be modulo(360). Thus an angle of $200^{\circ}$ may have a constant of $180^{\circ}$ added to it, and become not $380^{\circ}$ but $20^{\circ}$. To take another example, the angle of $10^{\circ}$ is closer to the angle of $300^{\circ}$ than it is to $200^{\circ}$. Clearly, the application of routine statistical methods to directional data could be disastrous.

Directional data arise naturally in the study of animal behaviour. Mardia (1972) gave examples which included the vanishing angles of 714 mallard ducks which had been released after being displaced by varying distances from their normal habitat, the orientations of 76 turtles after an experimental treatment, and the angles between the swimming directions of Daphnia and the plane of polarization of light. Moore (1980) has recently used directional statistics in studying the use of solar cues by the Savannah sparrow.

Many forms of data whose occurrence is periodic in time can fruitfully be regarded as directional. The time of day or time of year, or time in a biological cycle such as the menstrual cycle, are examples. When the time period is 1 day, $360^{\circ}$ corresponds to $24 \mathrm{~h} ; 360^{\circ} / 24$, or $15^{\circ}$, corresponds to 1 hour, and $15^{\circ} / 60$, or $0.25^{\circ}$, corresponds to $1 \mathrm{~min}$. If midnight is taken as the zero direction, then noon corresponds to $180^{\circ}$ and 10:35 p.m. corresponds to an angle of $338.75^{\circ}$. In the directional representation, one minute before midnight is quite close to one minute after midnight. A researcher might be interested in the hypothesis that the average times of day at which babies are delivered differ between two hospitals, and a transformation of the delivery times into directions might be a useful way of dealing with the problem that time of day is essentially a circular variable. Such a transformation was used by Mosteller and Tukey (1977) in an example concerning the heights and times of high tides in Honolulu. Tufte (1978) has presented evidence for the 
hypothesis that economic variables are manipulated in accordance with the four-year electoral cycle in the U.S.A. Here again, a transformation of times into directions might be appropriate. In this case, $1^{\circ}$ corresponds to just over 4 days out of the 1461 in the four-year cycle. Mardia (1972) gave other examples, including a directional representation of the month of onset of leukemia.

Directional data also arise in geology and geophysics; indeed, many advances in the statistical theory of directions (not least, that due to Fisher (1953)) have stemmed from the requirements of these sciences. Studies of the three-dimensional directional components of remnent magnetism in rock samples have provided evidence in support of the theory of continental drift (Irving, 1964).

The present paper shows that a widely applicable model of preference judgements represents individual differences as directions over a space of small dimensionality, and that directional statistics can fruitfully be used with such a representation. When the representation of preferences is in two dimensions, individual differences are reflected in differences between the locations of points on the circumference of a unit circle; or, equivalently, they are manifest as differences between angles in the interval $\left(0^{\circ}, 360^{\circ}\right)$. Two varieties of the vector model for representing preferences are described and data from a market research study are used in order to illustrate the application of statistical tests to these directional representations. The statistical theory of directional data has been developed for directions in two, three and higher dimensionalities, but this paper is confined largely to the two-dimensional case. The interested reader is referred to Mardia (1972, 1975) and Stephens (1962).

\section{Representation of Preferences as Vectors by a Factor-Analytic Method}

Social psychologists often obtain data in which each of many interviewees makes preference judgements about each of a standard set of stimuli. The factor-analytic procedure made available by Carroll and Chang (1964) and applied in market research by Green and Rao (1972) represents each individual's profile of preferences as a vector in a small number of dimensions. The method can be used with any set of profile scores: for example, those arising in repertory-grid studies (Bannister and Mair, 1968). It is often known as MDPREF, after the Carroll-Chang computer program. The factor-analytic decomposition of a rectangular matrix of preference judgements can be written as

$$
\mathrm{S}=\mathrm{G} X^{\prime}+\mathrm{E}
$$


where $\mathrm{S}$ has $n$ rows (for subjects) and $m$ columns (for stimuli) and contains the preference judgements as elements (the rows of $S$ should be centred), G has $n$ rows (for subjects) and $p$ columns, where $p$ is the number of factors the analyst wishes to deal with, $\mathrm{X}$ has $m$ rows (for stimuli) and $p$ columns and is called the "stimulus matrix", and E has $n$ rows and $m$ columns and its elements are the errors of prediction of $S$ from GX'.

The matrix $G$ is called the "subjects matrix". It is formed by norming column eigenvectors to their eigenvalues. The columns corresponding to the $p$ largest eigenvalues make up the columns of $\mathrm{G}$ and are mutually orthogonal. If the rows of $\mathrm{G}$ are normed to unity, their elements can be regarded as the direction cosines of the angles between a vector representing the $i$ th subject and the dimensions of the stimulus space defined by $\mathrm{X}$ (the vector passes through the centroid of $\mathrm{X}$ ). If $\mathrm{X}$ is taken as fixed, the rows of $\mathrm{G}$ can then be used as proxy information for the preference vectors which form the rows of $S$, since these are related linearly through $X$. It is possible to calculate the correlation between observed preferences and those predicted using the factor-analytic model for each subject. When these correlations indicate satisfactory goodness of fit and when $p$ is small in relation to the number of stimuli, it may be worthwhile to use $G$ as a summary version of $S$.

The vector-model representation compares sets of preference judgements by considering the stimuli (things judged) to be in a p-dimensional space and the subjects to be vectors passing through the origin of that space. The orientation of each subject vector is such as to maximize the correlation between the original preference judgements and the perpendicular projections of the stimuli onto the subject vector. The factor-analytic method determines the stimulus matrix $\mathrm{X}$ simultaneously with the orientations of the subject vectors, and is therefore called "internal analysis of preferences".

In the case when $p=2$ (a two-dimensional factor analysis of preferences), the orientation of each subject vector can be summarized as the angle 0 between the first dimension and the subject vector. The direction cosine with respect to the first factor in matrix $\mathrm{G}$ is then $\cos 0$ and that with respect to the second factor is $\sin 0$ (since the factors are orthogonal). The angle 0 may range from 0 to $360^{\circ}$.

\section{Representation of Preferences as Vectors by Embedding in an Externally Provided Stimulus Space}

In this approach, the coordinates of the stimuli upon a set of dimensions must be provided by the data analyst (this is in contrast to the factor-analytic method above, in which the stimulus coordinates are determined together with the individual subject vectors from the preferences data). The 
stimulus coordinates will usually be provided from a prior investigation, possibly with a different type of data (e.g., Green and Rao, 1972; Coxon and Jones, 1978). Multiple-regression methods are used to relate each preference profile to the stimulus coordinates, and after a norming step the set of regression coefficients for each profile can be interpreted as a vector (Kruskal and Wish, 1978). The method is known as "external analysis of preferences by a vector model" (Carroll, 1972) and is embodied in the PREFMAP computer program written by Carroll and Chang. Carroll's external analysis procedure consists of a number of related models, of which we are concerned with the fourth, or vector, mode

$$
S_{i j}=a_{i}+\sum_{m=1}^{m-p} b_{i m} X_{j m}+e_{i j}
$$

where $s_{i j}$ is the preference of the ith subject for the $j t h$ stimulus, $x_{j m}$ is the coordinate of the $j$ th stimulus on the $m$ th dimension, $a_{i}$ is an intercept term for the ith subject, bim is a partial regression coefficient for the ith subject on the mth dimension, and $e_{i j}$ is the error of prediction.

If the matrix $X$ of stimulus coordinates is taken as given, then the vector of preference judgements made by the $i$ th subject can be represented by the corresponding vector of partial regression coefficients. The adequacy of the representation depends on the error of prediction. The correlation between the observed and fitted preference values can be estimated for each subject. A useful degree of data reduction may be achieved if such correlations are large and the number of partial regression coefficients estimated for each subject $(p)$ is small in relation to the number of stimuli evaluated. The coefficients may be estimated by least-squares regression, or if it is desired to treat each subject's preferences as ordinal, by monotone regression (see Kruskal and Wish, 1978). In the latter case, the level of measurement of preferences is raised from ordinal to interval, since the regression coefficients are real numbers.

After each subject's vector of partial regression coefficients has been normed to unity, its elements can be regarded as direction cosines with' respect to the dimensions of the stimulus space $X$. In the same way as for internal analysis, two-dimensional analysis allows the summary of each vector of preferences by an angle () which indicates the orientation of the fitted subject vector with respect to the first dimension of the stimulus space. Again, the angles may range in the interval $0-360^{\circ}$.

It should be borne in mind that the vector model is by no means the only way of representing preference judgements. Carroll (1972) described four related models for this purpose, and the vector model is the simplest of these, the others being variants of the "ideal-point" model for preferences (Coombs, 1964). Good and Tideman (1976) have discussed this ideal-point 
model in the context of the aggregation of individual preferences into a collective choice. A goodness-of-fit correlation can always be calculated so as to check the extent to which the data of any subject are summarized adequately by the scaling representation. If this precaution is taken routinely, the vector model can be very useful in representing preferences. It may also be noticed that each subject's set of preferences over the whole set of stimuli is fitted as a unit, so that the scaling method keeps together the relational properties of a subject's judgements.

\section{Meaningfulness of the Directional Representation of Preferences}

There is no natural origin for the directions representing individual preferences over a set of stimuli, and therefore the angular locations of these directions must be measured either clockwise or counter-clockwise from some arbitrary reference point, which is usually taken as the positive direction of the first dimension of the stimulus matrix X. The measurement is conventionally made counter-clockwise, in the sense that the positive direction of the second dimension is at an angle of $+90^{\circ}$. Furthermore, the internal-analysis scaling solution is determined only up to an affine transformation, so that any translation, reflection, rotation or differential stretching of the stimulus matrix X is permitted (Green and Rao, 1972). Each of these transformations of the stimulus matrix requires a corresponding transformation of the directions representing individual subjects in order that the factor-analytic decomposition of the original data matrix remain valid. Thus the angles by which individual preferences are summarized have meaning only with reference to a particular origin of a particular transformation of the stimulus matrix $\mathrm{X}$. The differences between angles are invariant over choice of reference direction and over all rotations of the stimulus matrix. It follows that statistics based on differences between angles will be similarly invariant over rotations of $X$. Neither the absolute values of angles nor the differences between angles are preserved over differential stretchings of the axes of the stimulus matrix $\mathrm{X}$. Fortunately, the ratios of tangents of angles are invariant over such transformations, and therefore the rank order of the angles themselves must be similarly preserved. Hence statistical tests based on the ranks of the angles will be invariant over all permissible transformations of the solution to the factor-analytic decomposition of the original preferences data. As will be seen below, nonparametric tests for directional data have been developed only for the two-dimensional case, and therefore the above considerations limit attention to two-dimensional analysis of preferences. In internal analysis, the stimulus matrix itself is determined from the characteristics of the particular sample of data. If the set of all 
affine transformations of the same matrix is taken as an equivalence class, it can be argued that, conditional on dimensionality, there is only one solution that maximizes explained variation in a particular set of data, and that this solution is associated with a unique ordering of the angles representing preference vectors.

Much the same considerations of meaningfulness and uniqueness apply to the vector representation of preferences by external analysis. As is the case for internal analysis, differences between the directions of preference vectors are invariant over choice of reference direction and over all rotations of the stimulus matrix. The main difference from internal analysis is that the choice of the externally supplied stimulus matrix is entirely up to the researcher. Depending upon the theoretical considerations underlying the choice of such a stimulus matrix, it mayor may not make sense to allow the full set of affine transformations upon it. If the externally supplied stimulus matrix is determined only up to an affine transformation, exactly the same considerations apply as noted above for internal analysis. On the other hand, if differential stretchings of axes are not permitted, it is possible to use the differences between angles as data in carrying out statistical tests. One general point about external analysis is that the researcher is free to choose among stimulus matrices which need not be related by any transformation. It follows that statements about differences between, or even the order of, angles representing preference vectors are contingent on the particular stimulus matrix chosen by the investigator.

Another model which represents individual differences as directions is the weighted Euclidean distances model, which has been used mostly to analyze replicated judgements of the degree of similarity or dissimilarity between pairs of stimuli, and is due to Bloxom (1968), Horan (1969) and Carroll and Chang (1970). Each individual is characterized by a weight on each of the dimensions of a stimulus matrix, both the individual weights and the common stimulus matrix being estimated from the data by a method which maximizes explained variation over all subjects. There is little point in presenting the model here. Carroll and Chang showed that the relative of the dimension weights for each individual can be regarded as directional information. At first sight, this would suggest that such a representation of individual differences can be analyzed by methods of the kind discussed in this paper. However, since the dimension weights are almost always positive (to such an extent that negative weights are regarded as symptomatic of a poor solution), the directions corresponding to individuals are necessarily confined to the positive quadrant (assuming the simple case of two-dimensional analysis), and when measured in degrees are limited to the range $\left(0^{\circ}\right.$, $90^{\circ}$ ). Following some remarks by Batschelet (1975), there is little to be gained by treating such data by directional statistics. A further objection is 
that the set of permissible transformations of the solution to Carroll and Chang's widely used version of the weighted Euclidean distances model includes transformations under which neither angles representing ratios of subjects' weights over the various dimensions nor the differences between these angles are preserved. Thus the directional representation of individual differences in judgements of similarities or dissimilarities is contingent upon arbitrary normalizing conventions.

\section{Hypothesis Testing with Directional Representations of Preferences}

Having summarized complex evaluative judgements as directions, the data analyst may have hypotheses concerning subgroup differences which can now be expressed as hypotheses concerned with differences in the mean directions of sets of subject vectors. A dramatic illustration of such group differences (though without any attempt at statistical inference) was given by Wish (1971) in a study of evaluations of nations. Directional data can also arise in the form of paired vectors, the $i t h$ case having two properties, each of which is in a directional representation, and in such a case it is natural to require some index of correlation between the paired vectors. It may also be necessary to inquire about the correlation between a directional property and a linear variable. Following from considerations in the above discussion of the meaningfulness of directional representations of preferences, special consideration will be given to nonparametric statistical tests. Since a goodness-of-fit correlation is obtained for each set of preferences summarized as a direction, it is also desirable to test hypotheses concerning the determinants of differential fit to the vector model of preferences.

The data used in the present exemplary analyses are from Green and Rao (1972). A sample of 42 subjects (21 married couples) made preference judgements about 15 food items. The preferences data were rankings and are here treated as if they were real numbers. Furthermore, the sample was nonrandom and we shall assume random sampling. Nevertheless, the numbers serve well for illustrative purposes, since the basic data with details of the scaling methods by which they were analyzed were described fully by the authors.

Green and Rao reported the representation of preferences as directions by two methods; first using internal analysis (MDPREF) in two and three dimensions (Green and Rao, 1972, pp. 244-245), and second using external analysis (PREFMAP vector model) in only two dimensions (Green and Rao, 1972, p. 260). External analysis was carried out using different stimulus matrices in two subgroups of the 42 subjects. The authors reported two-dimensional plots (p. 85, pp. 118-9) which give some indication of the shape 
TABLE I

Two-Dimensional Representation of Preferences as Directions ${ }^{\text {a }}$

\begin{tabular}{|c|c|c|c|c|c|c|}
\hline Case & $\theta$ (deg.) & $\cos \theta$ & $\sin \theta$ & Rank & $r$ & OVWT \\
\hline 1 & 180.0 & -1.0000 & 0.0060 & 14 & 0.77 & 1 \\
\hline 2 & 175.0 & -0.9962 & 0.0868 & 18 & 0.91 & 0 \\
\hline 3 & 148.3 & -0.8508 & 0.5254 & 24 & 0.65 & 0 \\
\hline 4 & 205.5 & -0.9025 & -0.4307 & 5 & 0.94 & 1 \\
\hline 5 & 131.9 & -0.6676 & 0.7445 & 28 & 0.72 & 1 \\
\hline 6 & 194.5 & -0.9680 & -0.2510 & 9 & 0.92 & 1 \\
\hline 7 & 178.6 & -0.9997 & 0.0252 & 15 & 0.88 & 0 \\
\hline 8 & 175.3 & -0.9966 & 0.0821 & 17 & 0.86 & 0 \\
\hline 9 & 155.7 & -0.9112 & 0.4120 & 22 & 0.67 & 1 \\
\hline 10 & 153.5 & -0.8950 & 0.4461 & 23 & 0.85 & 0 \\
\hline 11 & 38.0 & 0.7875 & 0.6163 & 40 & 0.94 & 0 \\
\hline 12 & 74.7 & 0.2633 & 0.9647 & 35 & 0.80 & 0 \\
\hline 13 & 163.9 & -0.9606 & 0.2781 & 21 & 0.32 & 0 \\
\hline 14 & 185.1 & -0.9961 & -0.0878 & 12 & 0.76 & 1 \\
\hline 15 & 185.4 & -0.9956 & -0.0940 & 11 & 0.89 & 0 \\
\hline 16 & 139.4 & -0.7588 & 0.6513 & 25 & 0.80 & 0 \\
\hline 17 & 196.9 & -0.9570 & -0.2902 & 7 & 0.84 & 0 \\
\hline 18 & 166.7 & -0.9733 & 0.2294 & 20 & 0.72 & 0 \\
\hline 19 & 252.8 & -0.2962 & -0.9551 & 4 & 0.64 & 1 \\
\hline 20 & 200.4 & -0.9371 & -0.3490 & 6 & 0.65 & 0 \\
\hline 21 & 47.0 & 0.6824 & 0.7309 & 38 & 0.32 & 1 \\
\hline 22 & 187.6 & -0.9912 & -0.1323 & 10 & 0.90 & 1 \\
\hline 23 & 115.2 & -0.4251 & 0.9052 & 30 & 0.74 & 1 \\
\hline 24 & 116.7 & -0.4911 & 0.8711 & 29 & 0.52 & 0 \\
\hline 25 & 171.9 & -0.9901 & 0.1400 & 19 & 0.53 & 0 \\
\hline 26 & 177.9 & -0.9993 & 0.0384 & 16 & 0.85 & 0 \\
\hline 27 & 36.1 & 0.8076 & 0.5898 & 41 & 0.72 & 0 \\
\hline 28 & 138.5 & -0.7485 & 0.6631 & 26 & 0.66 & 0 \\
\hline 29 & 196.3 & -0.9598 & -0.2807 & 8 & 0.59 & 1 \\
\hline 30 & 183.5 & -0.9981 & -0.0616 & 13 & 0.89 & 0 \\
\hline 31 & 93.9 & -0.0688 & 0.9976 & 33 & 0.33 & 0 \\
\hline 32 & 3.6 & 0.9980 & 0.0628 & 42 & 0.48 & 0 \\
\hline 33 & 108.6 & -0.3194 & 0.9476 & 32 & 0.79 & 1 \\
\hline 34 & 112.4 & -0.3807 & 0.9247 & 31 & 0.80 & 1 \\
\hline 35 & 66.0 & 0.4067 & 0.9135 & 36 & 0.48 & 1 \\
\hline 36 & 352.7 & 0.9918 & -0.1281 & 1 & 0.33 & 1 \\
\hline 37 & 346.4 & 0.9719 & -0.2352 & 2 & 0.65 & 1 \\
\hline 38 & 137.1 & -0.7322 & 0.6811 & 27 & 0.70 & 1 \\
\hline 39 & 310.9 & 0.6542 & -0.7563 & 3 & 0.66 & 1 \\
\hline 40 & 46.6 & 0.6875 & 0.7262 & 39 & 0.90 & 1 \\
\hline 41 & 77.2 & 0.2219 & 0.9751 & 34 & 0.74 & 0 \\
\hline 42 & 59.9 & 0.5008 & 0.8656 & 37 & 0.73 & 0 \\
\hline \multicolumn{7}{|c|}{$C=\sum \cos \theta_{i}=-16.18$} \\
\hline \multicolumn{7}{|c|}{$S=\sum^{i} \sin \theta_{i}=12.03$} \\
\hline \multicolumn{7}{|c|}{$R^{2}={ }^{i} C^{2}+S^{2}=406.6$} \\
\hline \multicolumn{7}{|c|}{$R=20.165$} \\
\hline \multicolumn{7}{|c|}{$R / n=0.48$} \\
\hline
\end{tabular}

a The scaling results in this table are taken from an analysis reported by Green and Rao (1972). 
of the angular distribution of the subject vectors summarizing the individual preference rankings. They also presented background data on such characteristics as age, sex and whether or not the subject was overweight. Thus their data can be used to illustrate appropriate methods for handling directional data of the type which can arise in individual-differences scaling of preferences.

The angles indicating the orientations of the subject vectors from internal scaling of preferences are shown in Table 1. These angles were obtained using the methods outlined above, using standard trigonometry on the results reported by Green and Rao. The median of the goodness-of-fit correlations over the 42 subjects is 0.75 , which is reasonable for a two-dimensional analysis. Results for the three-dimensional internal analysis and the two-dimensional external analysis are not presented here.

Descriptive and Inferential Statistics for Directions in Two Dimensions

This paper does not attempt a complete review of the statistics of directions. The interested reader is referred to Mardia $(1972,1975)$ and to Pearson and Hartley (1972, pp. 123-133). Some basic concepts and techniques are instead outlined and illustrated using the Green and Rao data.

(a) Each observation (direction) is a vector of unit length, and in the most general case may be thought of as a point on the surface of a unit hypersphere in $p$ dimensions. In two dimensions, each observation is regarded as a point on the circumference of a unit circle and the Cartesian coordinates of that point are given as $\cos \theta, \sin \theta$, where $\theta$ is the angular observation of interest.

(b) The mean direction is defined as the direction of the resultant vector of all the vectors in the sample (the resultant vector being simply the vector sum). This is illustrated in Table I, which shows the computation of the mean direction for the vectors from a two-dimensional internal analysis of preferences. The sums of $\cos \theta_{i}$ and $\sin \theta_{i}$ over the 42 cases are -16.18 and 12.03 , respectively, and the length of the resultant vector is given by Pythagoras' theorem as 20.16. Standard trigonometry indicates that this corresponds to an angle of $180^{\circ}-36.64^{\circ}$, or $143.36^{\circ}$.

(c) The notation used by Mardia (1972) for the two-dimensional case is helpful, and is as follows. Given a sample of angles $\theta_{i}$ of size $n$, where

$$
C=\sum_{i=1}^{i=n} \cos \theta_{i}
$$


and

$S=\sum_{i=1}^{i=n} \sin \theta_{i}$

the length of the resultant vector is given by

$$
R=\left(C^{2}+S^{2}\right)^{1 / 2}
$$

Dividing the quantities $C, S$ and $R$ by $n$ yields $\bar{C}, \bar{S}$ and $\bar{R}$; the mean direction $\bar{x}_{0}$ can then be obtained by evaluating the two equations

$$
C=\bar{R} \cos \bar{x}_{0}
$$

and

$$
\bar{S}=\vec{R} \sin \bar{x}_{0}
$$

(d) The quantities $C, S$ and $R$ can be computed for the total sample of cases and also for subsamples, in which case they can be given appropriate subscripts, for example $C_{j}, S_{j}$ and $R_{j}$. The lengths $R$ or $R_{j}$ of resultant vectors turn out to be of recurring importance in tests of statistical significance for directional data. This is not surprising when it is realized that the length of a resultant vector is a measure of the extent to which its constituent individual vectors are spread out, or alternatively, is an inverse measure of the dispersion of the angles $\theta_{i}$ about their mean direction $\bar{x}_{0}$. Mardia (1972) defined a measure of dispersion as

$$
S_{0}=1-\cos \left(\theta_{i}-\bar{x}_{0}\right) / n
$$

Mardia also showed that $S_{0}=1-\bar{R}$, where $\bar{R}=R / n$. It follows that $R$ depends only on differences between angles.

\section{A Nonparametric Two-Sample Test for Circular Data}

The uniform-scores test, originally proposed by Wheeler and Watson (1964), is a convenient distribution-free two-sample test. Given directional data which can be considered as points on the circumference of a circle, two samples of observations (sizes $n_{1}$ and $n_{2}$ ) can be combined, and the overall batch of angles $\theta_{i}$ can be ranked in order of increasing size, on moving clockwise or anti-clockwise from any reference point. Ties may be broken randomly if necessary. Nonparametric tests develop statistics based on these ranks. In particular, the ranks of the first sample, denoted $r_{1}, \ldots, r_{n_{1}}$, are all that is needed to carry out the uniform-scores test.

After the observations for the combined sample have been ordered, the ranks can be multiplied by $360 / n$ (where $n$ is $n_{1}+n_{2}$ ), a procedure which 
turns the ranks into a uniformly distributed set of angles in the same order as the original data. The resultant vector from a uniformly distributed set of angles has zero length $(R=0)$, a point which can easily be seen by considering the resultant of the four angles $0^{\circ}, 90^{\circ}, 180^{\circ}$ and $270^{\circ}$. The uniform scores for the two subsamples can be recovered from the combined sample, and sums of cosines, sums of sines and the resultant lengths can be calculated. The sum of $C_{1}$ and $C_{2}$ must be zero, as must the sum of $S_{1}$ and $S_{2}$, while the lengths of $\mathbf{R}_{1}$ and $\mathbf{R}_{2}$ must be equal. The test statistic for the Wheeler and Watson test is based on the resultant length of the uniform scores for either of the two samples. The uniform scores are defined as 360 $r_{i} / n$, where $n=n_{1}+n_{2}$ and the $r_{i}$ are the ranks of the observations in the combined sample.

Then

$$
C_{1}=\sum_{i=1}^{i=n_{1}} \cos \left(360 r_{i} / n\right)
$$

and

$$
S_{1}=\sum_{i=1}^{i=n_{1}} \sin \left(360 r_{i} / n\right)
$$

with

$$
R_{1}^{2}=C_{1}^{2}+S_{1}^{2}
$$

The greater the separation between the points of the first sample and those of the second, the greater the lengths of the resultant vectors $\mathbf{R}_{1}$ and $\mathbf{R}_{2}$. Mardia gave a table of critical values for the test statistic $B=R_{1}^{2}$, and also showed that for $n$ greater than 20 , the quantity

$R^{*}=2(n-1) R_{1}^{2} / n_{1} n_{2}$

is distributed as $\chi^{2}$ with 2 degrees of freedom when the null hypothesis is true.

\section{Example I}

The directional data resulting from an internal analysis of preferences for breakfast foods might reasonably be expected to be related to a classification of subjects as "overweight" versus "not overweight". Indeed, Green and Rao (1972, p. 103) noted the presence of a correlation between excess weight and preference for sweet items, though the correlation was "not compellingly high". 


\section{TABLE II}

Calculations for Wheeler-Watson Two-Sample Test on Rank Data from Subsample of 19 Overweight Subjects

\begin{tabular}{llll}
\hline $\begin{array}{l}\text { Rank } \\
\left(r_{i}\right)\end{array}$ & $\begin{array}{l}\text { Angle } \\
\left(360 r_{i} / n\right)\end{array}$ & $\begin{array}{l}\text { cos } \\
\left(360 r_{i} / n\right)\end{array}$ & $\begin{array}{l}\text { sin } \\
\left(360 r_{i} / n\right)\end{array}$ \\
\hline 1 & 8.6 & 0.989 & 0.149 \\
2 & 17.1 & 0.956 & 0.295 \\
3 & 25.7 & 0.901 & 0.434 \\
4 & 34.3 & 0.826 & 0.563 \\
5 & 42.9 & 0.733 & 0.680 \\
8 & 68.6 & 0.365 & 0.931 \\
9 & 77.1 & 0.223 & 0.975 \\
10 & 85.7 & 0.075 & 0.997 \\
12 & 102.9 & -0.223 & 0.975 \\
14 & 120.0 & -0.500 & 0.866 \\
22 & 188.6 & -0.989 & -0.149 \\
27 & 231.4 & -0.624 & -0.782 \\
28 & 240.0 & -0.500 & -0.866 \\
30 & 257.1 & -0.223 & -0.975 \\
31 & 265.7 & -0.075 & -0.997 \\
32 & 274.3 & 0.075 & -0.997 \\
36 & 308.6 & 0.623 & -0.782 \\
38 & 325.7 & 0.826 & -0.563 \\
39 & 334.3 & 0.901 & -0.434 \\
Sums & & 4.359 & 0.320 \\
\hline
\end{tabular}

Taking the "overweight" group, since it has the smaller number of cases $(n=19)$, the results of Table II yield $C_{1}=4.36, S_{1}=0.32$ and $R_{1}^{2}=19.10$, which corresponds to $R^{*}=3.58$, which is significant only at the $20 \%$ level (by comparison with tables of $\chi^{2}$ at 2 d.f.).

Mardia (1972) also discussed extensions of the uniform-scores test to the multisample case.

\section{Mardia's Circular Rank Correlation Coefficient}

Given $\theta_{i}$ and $\phi_{i}$ as paired angular variables indicating directions in two-dimensional space, it is natural to seek an appropriate correlation coefficient. When working with data on the line, two variables are perfectly correlated when one is a linear transformation of the other. By analogy, it can be argued that directional data are perfectly correlated when the rotation 
their paired vectors. Mardia (1975) put this as follows:

$\theta=(\psi+\phi) \bmod (360)$

or

$\theta=(\psi-\phi) \bmod (360)$

Suppose that $\psi$ is $90^{\circ}$ and $\theta$ is $10^{\circ}, 20^{\circ}, 30^{\circ}$ for three cases. Then under condition (a) $\phi$ is $280^{\circ}, 290^{\circ}, 300^{\circ}$, while under condition (b) $\phi$ is $80^{\circ}, 70^{\circ}$, $60^{\circ}$.

Considering the problem as one of rank correlation evades the issue of estimating the angle of rotation $\psi$, and in fact the procedure is much the same as for the uniform-scores test, replacing the angles $\theta_{i}$ and $\phi_{i}$ by their ranks from 1 to $n$, and transforming these to the uniformly distributed scores by multiplying by $360 / n$. If (a) is true, then the mean resultant length of the differences between paired uniform scores should be unity. If (b) is true, then the same should be the case for the mean resultant length of the sums of paired uniform scores. Mardia's rank correlation $r_{0}$ is defined as the larger of the two squared mean resultant lengths, or

$$
r_{0}=\max \left(\vec{R}_{1}^{2}, \vec{R}_{2}^{2}\right)
$$

where $\bar{R}_{1}$ is the mean resultant length of the differences between corresponding uniform scores $\theta_{i}$ and $\phi_{i}$ and $\bar{R}_{2}$ is the mean resultant length of the sums of corresponding uniform scores $\theta_{i}$ and $\phi_{i}\left(r_{0}\right.$ varies between zero and unity).

Mardia showed that for large $n$, the critical value of $r_{0}$ for significance at level $\alpha$ is

$r_{0 \alpha}=-(n-1)^{-1} \log _{e}\left[1-(1-\alpha)^{1 / 2}\right]$

For further details, see Mardia (1975).

\section{Example II}

Since the Green and Rao data came from 21 married couples, it is possible to consider the correlation of preferences between spouses. It is convenient to use the angular representation derived from internal analysis of the subjects' preferences for breakfast-food items, i.e., from two-dimensional MDPREF analysis. The data of Table I are arranged with couples together, the first member of each couple being male and the second female.

The husband-wife correlation is 0.22 , being the larger of the two quantities $\vec{R}_{1}^{2}=0.10$ and $\vec{R}_{2}^{2}=0.22$. This exceeds the critical value of $r_{0}$ at the $5 \%$ level for 21 cases, and therefore the null hypothesis of independence of preferences can be rejected. The fact that it is the second mean-squared 
resultant which is the larger means that the circular rank correlation is negative; i.e., the circular ordering of preference vectors for men is to some degree the inverse of the ordering for their partners. Note that this refers to the circular ordering of the vectors representing the preferences, and not to the preference orderings themselves.

\section{Tests based on the Von Mises-Fisher Distribution}

The statistical theory developed by Von Mises (1918) and by Fisher (1953), and extended by Mardia (1975), Stephens (1962) and Watson and Williams (1956), considers the distribution of points on the surface of a hypersphere in $p$ dimensions. The Von Mises-Fisher distribution is symmetrical about a polar axis and attains a maximum density at the pole and a minimum density at the anti-pole. In two dimensions it is known as the Von Mises distribution and in three dimensions as the Fisher distribution. Being conceptually simple and mathematically tractable, the Von Mises-Fisher distribution is in some ways the analogue of the normal distribution in conventional statistics. It has an important parameter, $\kappa$, which determines the clustering of the distribution of points around the polar axis (or equivalently, the degree of clustering of the distribution of the vectors in $p$-dimensional space). High values of $\kappa$ generate a concentrated distribution around the polar axis, while a zero value would generate a uniform distribution over the surface of the $p$-dimensional hypersphere. The notion of a uniform distribution of angles around the circle has been discussed already above. A number of results in the statistical theory of directional data depend on the value of $\kappa$ that is assumed to characterize a population distribution. Fortunately, it is possible to obtain a maximum-likelihood estimate of $\kappa$ from sample data, since it is related to the mean resultant length (Mardia, 1972, pp. 250-251).

The relationships between the Von Mises distribution and other distributions on the circle have been discussed in detail by Mardia (1972). It is worth noting that the Von Mises and the wrapped normal distributions can be made to approximate each other closely (a wrapped distribution is generated from the corresponding function on the line by transforming the "linear" random variables modulo $(2 \pi)$ ).

\section{The Watson-Williams Test}

Watson (1956) and Watson and Williams (1956) proposed an approximate multisample test for the equality of mean vectors. The test is somewhat 
analogous to one-way analysis of variance, and while it was originally developed for distributions on the circle and on the sphere, it has been generalized to the $p$-dimensional case. Following the notation of Mardia (1972), the total of $n$ cases is considered as being divided into $q$ samples, the length of the overall resultant vector being $R$, while the length of the resultant vector in the $i$ th sample is $R_{i}$. A useful test statistic is then given by

$$
U=(n-q)\left(\sum R_{i}-R\right) /(q-1)\left(n-\sum R_{i}\right)
$$

In the two-dimensional case, $U$ is distributed approximately as an $F$ distribution with $q-1$ and $n-q$ degrees of freedom. In three dimensions, the degrees of freedom are $2 q-2$ and $2 n-2 q$, and in $p$ dimensions they are $(p-1)(q-1)$ and $(p-1)(n-q)$ (see Stephens $(1962))$. When the test statistic is larger than the critical value of $F$ at the appropriate number of degrees of freedom, it can be concluded that the subsamples do not come from the same population.

The above analysis can be set up in the usual tabular scheme for one-way analysis of variance, with the "between-groups" sum of squares being estimated as $\sum R_{i}-R$ and the "within-groups" sum of squares as $n-\Sigma R_{i}$, the total being given as $n-R$ (strictly speaking, each of these components should be multiplied by twice the value of $\kappa$ but since ratios are used for the tests, this step may be omitted):

\section{Variance components}

Between-groups

Within-groups

Total

$$
\sum_{i} R_{i}-R
$$$$
n-\sum_{i} R_{i}
$$$$
n-R
$$

d.f.

$$
\begin{aligned}
& (q-1)(p-1) \\
& (n-q)(p-1) \\
& (n-1)(p-1)
\end{aligned}
$$

The test assumes that the vectors come from a population distributed according to the $p$-dimensional Von Mises distribution, and that the concentration of vectors in all samples is the same. Unfortunately, the simple formula for the test statistic $U$ depends upon an approximation which holds true only for large values of $\kappa$. Mardia (1972) cited Monte Carlo studies by Stephens which showed that the simple formula for $U$ can be used uncorrected in two dimensions if the overall mean resultant length exceeds 0.95 , and can be used uncorrected in three dimensions if the mean resultant length exceeds 0.67 , these cutting points corresponding to $\kappa$ values of 10.0 in two dimensions and 3.0 in three dimensions. The correction formulae contain estimates of $\kappa$ as derived from the mean resultant length from tables given by 
Mardia (1972):

Dimensions

Two

Two

Three

Three
Mean resultant length

$$
\begin{array}{r}
0.45<R / n<0.95 \\
R / n<0.45 \\
0.32<R / n<0.67 \\
R / n<0.32
\end{array}
$$

Correction by which

$U$ is multiplied

$1+3 / 8 k$

$1 /\left(1-k^{2} / 8+q / 2 n k^{2}\right)$

$1-1 / 5 k^{2}$

$1 /\left(1-k^{2} / 15+3 q / 2 n k^{2}\right)$

where $k$ is the estimate of $\kappa, q$ is the number of samples, and $n$ is the total number of vectors. The $k$ values in the above formulae are maximum-likelihood estimates of the population value. Correction formulae for higher dimensionalities have not been derived.

No examples of the application of the Watson-Williams test to directional representations of preferences are given here, since it has been shown above that internal analysis yields only the circular rank order of the directions as a summary which is invariant over the permissible transformations of the scaling solution, while the external analyses of the data from Green and Rao fit the data rather poorly. No useful nonparametric tests have been developed for the three or higher dimensional case.

\section{Discussion}

The statistics of directions are useful in studies of animal behaviour and of periodic phenomena. This paper has examined the conditions under which directional representations of preferences can meaningfully be analyzed adopting such an approach. It has been shown that given the indeterminacy of the multidimensional scaling solutions which are used to represent preference vectors as directions, the only defensible strategy is to restrict analysis to a two-dimensional scaling and to use nonparametric tests developed in directional statistics on the rank order of the angular representation of preferences around the circle. When preferences data are transformed into directions by an external analysis procedure, it may sometimes be permissible to use parametric directional statistics, though this will depend first on whether or not it is thought that the axes of the externally supplied stimulus matrix are subject to differential stretching, and secondly on whether or not the distributional assumptions of the Von Mises-Fisher distribution are satisfied. Interesting work has recently been reported on the definition of various indices of correlation for directional data (Stephens, 1969; Jupp and Mardia, 1980). However, the conservative approach to the analysis of 
preferences using these methods is probably to use the nonparametric tests discussed above.

The scaling method that represents each subject's set of preferences over the whole set of stimuli as a direction on a circle is appealing because it keeps together the relational properties of many judgements by the same person (for example, that toast was most preferred and that ,blueberry muffin was least preferred). A two-dimensional representation of preferences according to the vector model will not always be possible, since the explained variation may be inadequate. However, on occasions when this way of looking at preferences data is appropriate, there are special nonparametric tests for the significance of differences in directions between two or more samples, and for the correlation between paired angular data.

\section{Acknowledgement}

The research reported in this paper was supported by SSHRC grant no. 410-77-0860.

\section{References}

Bannister, D. and Mair, J.M.M. (1968). The Psychology of Personal Constructs. New York: Academic Press.

Batschelet, E. (1975). "Contribution to discussion of paper by K,V. Mardia", Journal of the Royal Statistical Society, B Series 37: 378.

Bloxom, B. (1968). "Individual differences in multidimensional scaling", Educational Testing Service (Princeton, NJ), Research Bulletin 68-45.

Carroll, J.D. (1972). "Individual differences and multidimensional scaling", pp. 105-155 in R.N. Shepard, AK, Romney and S.B. Nerlove, eds., Multidimensional Scaling: Theory and Applications in the Behavioural Sciences. Vol. 1. New York: Seminar Press.

Carroll, J.D. and Chang, J.J. (1964). "Nonparametric multidimensional analysis of paired comparisons data", unpublished paper, Bell Laboratories, Murray Hill, NJ.

Carroll, J.D. and Chang, J.J, (1970). "Analysis of individual differences in multidimensional scaling via an N-way generalization of the' Eckart-Young' decomposition", Psychometrika 35: 238-319.

Coombs, C.H. (1964). A Theory of Data. New York: Wiley.

Coxon, AP.M. and Jones, C.L. (1978). The Images of Occupational Prestige. London: Macmillan.

Fisher, R.A (1953). "Dispersion on a sphere", Proceedings of the Royal Society (London), Series A 347: 371-385.

Good, I.J. and Tideman, T.N. (1976). "From individual to collective ordering through multidimensional attribute space", Proceedings of the Royal Society (London), Series A 347: 371-385.

Green, P.E. and Rao, V.R. (1972). Applied Multidimensional Scaling. New York: Holt, Rinehart and Winston. 
Horan, C.B. (1969). "Multidimensional scaling: combining observations when individuals have different perceptual structures", Psychometrika 34: 139-165.

Irving, E. (1964). Palaeomagnetism and its Application to Geological and Geophysical Problems. New York: Wiley.

Jupp, P.E. and Mardia, K.V. (1980). "A general correlation coefficient for directional data and related regression problems", Biometrika 67: 163-173.

Kruskal, J.B. and Wish, M. (1978). Multidimensional Scaling. Beverly Hills, CA: Sage.

Mardia, K.V. (1972). Statistics of Directional Data. New York: Academic Press.

Mardia, K.V. (1975). "Statistics of directional data (with discussion)", Journal of the Royal Statistical Society, B Series 37: 349-393.

Moore, F.R. (1980). "Solar cues in the migratory orientation of the Savannah sparrow", Animal Behaviour 28: 684-704.

Mosteller, F. and Tukey, J.W. (1977). Data Analysis and Regression. Reading, MA: Addison-Wesley.

Pearson, E.S. and Hartley, H.O., eds. (1972). Biometrika Tables for Statisticians. Vol. Il. Cambridge: Cambridge University Press.

Stephens, M.A. (1962). "The statistics of directions", Ph.D. Thesis, University of Toronto.

Stephens, M.A. (1969). "Multi-sample tests for the Fisher distribution for directions", Biometrika 56: 169-181.

Tufte, E.R. (1978). Political Control of the Economy. Princeton, NJ: Princeton University Press.

Von Mises, R. (1918). "Uber die 'Ganzzahligkeit' der Atomgewicht und verwandte Fragen", Physikalische Zeitung, 19: 490-500.

Watson, G.S. (1956). "Analysis of dispersion on a sphere", Monthly Notices of the Royal Astronomical Society, Geophysical Supplement 7: 153-159.

Watson, G.S. and Williams, EJ. (1956). "On the construction of significance tests on the circle and the sphere", Biometrika 43: 344-352.

Wheeler, S. and Watson, G.S. (1964). "A distribution-free two-sample test on a circle", Biometrika 51: 256-257.

Wish, M. (1971). "Individual differences in perceptions and preferences among nations", pp. 312-327 in C.W. King and D. Tigert, eds., Attitude Research Reaches New Heights. Chicago: American Marketing Association. 